\title{
Energetics and kinetics of monolayer formation in vapor-liquid-solid nanowire growth
}

\author{
Frank Glas \\ Université Paris-Saclay, CNRS, Centre de Nanosciences et de Nanotechnologies, 91120 Palaiseau, France* \\ Vladimir G. Dubrovskii \\ ITMO University, Kronverkskiy pr. 49, 197101 Saint Petersburg, Russia ${ }^{\dagger}$
}

(Dated: May 20, 2020)

\begin{abstract}
The vapor-liquid-solid growth of semiconductor nanowires proceeds via the sequential addition of individual atomic or biatomic monolayers at the interface between the nanowire stem and a liquid catalyst droplet. Each monolayer growth cycle comprises a period of nucleation and extension of the monolayer followed by a waiting time before the next nucleation. The cyclic evolution of the liquid-solid system and the duration of these periods affect the properties of individual nanowires and the statistics of nanowire ensembles. Based on recent in situ growth experiments, we investigate theoretically the thermodynamics and kinetics of monolayer formation, with emphasis on nanowires of III-V materials. We first study the thermodynamics of the process in a quasistatic approximation. We calculate and minimize the work of formation of a partial monolayer, accounting for the depletion and refill of the liquid phase and all relevant interface energies. We focus on the regime where the liquid does not contain enough nanowire material at nucleation to form a full monolayer and where growth comprises a fast stage where a partial monolayer forms rapidly using the available material and a slower stage during droplet refill. We explore the effect of the non-monotonous variation of the total monolayer edge energy on these two growth stages and determine the partition between liquid and monolayer of the atoms provided during refill. We then develop a kinetic model that accounts in simple terms for the competition between delivery and attachment of atoms to the monolayer and droplet refill. We perform fully analytical calculations that describe a continuum between the pulsed regime explored in our thermodynamic study and a smooth regime where the monolayer extends at the pace of refill. The various characteristic times of the monolayer cycle are calculated in presence of desorption of the volatile group V species from the liquid droplet. Simplified asymptotic formulas corresponding to fast attachment or low desorption are derived. Desorption is found to affect weakly the monolayer formation time but strongly the waiting time.
\end{abstract}

\section{INTRODUCTION}

The nucleation and growth of a new phase from a supersaturated parent phase has been studied for decades. However, the development of nanostructures has revealed that, when the parent phase has nanometric dimensions, nucleation may present very original features. Although nanosize phases occur in many systems [1-6], their indepth study is only beginning, based on experimental advances and theoretical developments, .

A striking effect is that nucleation, followed by the rapid growth of the new phase, may significantly deplete the nanosized parent phase and hence alter the subsequent growth kinetics, if not nucleation itself. There is already a large body of theoretical and computational work about this kind of depletion effect, mainly insofar as it affects nucleation (or even prevents it, a phenomenon known as superstabilization) [7-12].

The vapor-liquid-solid (VLS) growth of semiconductor nanowires (NWs) is a choice system for investigating such effects, not least because theory can now be confronted to

\footnotetext{
* https://www.c2n.universite-paris-saclay.fr; frank.glas@c2n.upsaclay.fr

$\dagger$ dubrovskii@mail.ioffe.ru
}

experimental observations carried out in real time and at high spatial resolution in dedicated electron microscopes. Such in situ experiments confirm that NWs grow monolayer (ML) by ML [13-17], each ML starting via a single nucleation event occurring at the interface between the already formed solid NW stem and the apical liquid nanodroplet [15]. Recall that stem, ML and droplet have typical radii ranging from a few $\mathrm{nm}$ to some tens of $\mathrm{nm}$.

In the particular case of NWs of III-V compounds, the volatile group $\mathrm{V}$ element is present at very low concentration (on the order of $1 \%$ or less) in the liquid catalyst nanodroplet. This is true whether the liquid involves an extraneous metal (such as gold [18]) or not (self-catalyzed growth [19]). In III-V NWs, forming even a single ML may then significantly deplete the droplet in group $\mathrm{V}$ atoms. We demonstrated experimentally and theoretically that this has a strong impact on the statistics of nucleation $[2,20,21]$ : depletion makes a new nucleation less likely after a first one than before, so that the nucleation events are anti-correlated in time. This nucleation antibunching (which may also yield NW ensembles with very uniform lengths [22-24]) consists in the depletion induced by the fast growth of a ML affecting the formation of subsequent MLs. In addition, at a finer temporal and spatial level, Dubrovskii has recently considered theoretically how continuous depletion may affect the growth of each ML, independently of the others, possibly resulting 
in fractional MLs than cannot get completed before the vapor fluxes refill the liquid phase [25].

Quite generally, mononuclear NW growth can be considered as a series of ML cycles, each composed of three successive stages [26]: (i) nucleation of a new ML; (ii) extension of this ML by step flow until it covers entirely the solid-liquid interface; (iii) waiting time before next ML nucleates. Experimentally, nucleation is too elusive to be observed and, for III-V NWs, our experiments [15] lead us to describe the ML cycle as a succession of three observable stages (Fig. 1): (1) nucleation of a new ML and its fast extension using group $\mathrm{V}$ atoms available in the droplet, which may only suffice to cover partially the interface; (2) slow extension of the ML up to full interface coverage, now using group $\mathrm{V}$ atoms brought by the external vapor that refills the droplet; (3) waiting time before nucleation of the next ML, during which all newly provided atoms enrich the liquid. This applies for intermediate values of the droplet contact angle, for which the solid-liquid interface is not facetted $[14,17]$, whereas at large and small angles, ML extension may be fed by atoms from the already grown NW stem. Since nucleation is a stochastic process, the duration of these three stages varies from ML to ML, even if the average growth rate of the NW is fixed. Only stage 3 coincides with stage (iii) of the first description.

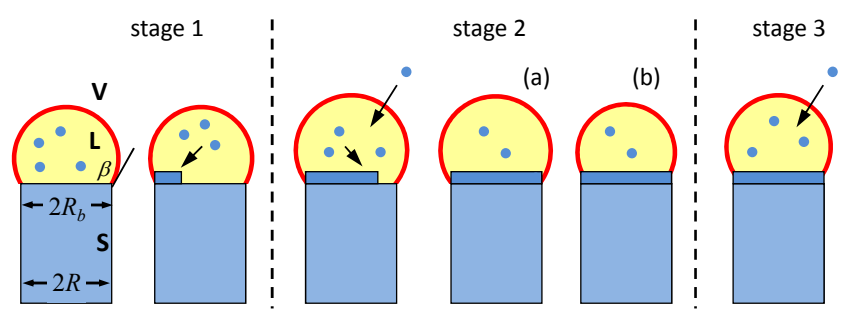

FIG. 1. Schematics of the three stages of the formation of a ML. The thick red line marks the external droplet area, taken into account in the energy calculations.

In our previous studies of nucleation antibunching, which did not include real time observations but only ex situ measurements, we assumed that the duration of stage 2 was very short compared with the interval between successive nucleations $[2,20,22]$. This requires in particular enough group $\mathrm{V}$ atoms in the droplet to complete rapidly the solid ML after its nucleation (in other words, stage 1 effectively covers the entire ML growth). However, other regimes are conceivable. One such regime occurs when the number of atoms available in the liquid at nucleation is even less than the group $\mathrm{V}$ content of a ML. This regime is now commonly observed in our molecular beam epitaxy (MBE) experiments [15]. In this case, the coverage of the solid/liquid interface by the new ML first increases abruptly (stage 1) and then much more slowly, so that stage 2 actually occurs, with a finite duration; see Figure 3 in ref. [15]. This is interpreted in the following way: the abrupt increase corresponds to the formation of the critical nucleus (typically only a few III-V pairs) and the rapid formation of a partial ML by using the As atoms available in the droplet. Subsequent ML extension is limited by the relatively slow refill of the droplet by the external fluxes. Yet another regime might occur in some chemical vapor deposition experiments, where the ML step flow appears as continuous, without any fast stage 1 (Ref. [14] and possibly Ref. [16]).

The present theoretical work considers these various growth regimes. It comprises two main parts. In the first part (Sections II to V), after introducing the energetics of the system in Section II, we study in detail the thermodynamics of ML formation in the case where a fast stage 1 (Section III) can be distinguished from a slower stage 2 (Section IV). We address the following questions. What is the fractional coverage achieved at the end of stage 1, depending on the state of the liquid droplet at nucleation and on the properties of the solid ML, in particular its edge energy? At stage 2, what is the partition of the newly provided atoms between incomplete ML and liquid, and therefore what is the rate of extension of the ML? However, such a time scale hierarchy [26] between stages 1 and 2 only exists if the kinetics of delivery of new pairs from the liquid and attachment to the ML (after nucleation) are fast with respect to the refill. In Sections III and IV, we ignore these kinetics: ML growth is treated as a quasistatic process, and we find the evolution of the state of the liquid-ML system by minimizing the formation energy of the ML. At stage 1, this only depends on the properties of the system, which are considered in detail, and on its pre-nucleation state. At stage 2, refill of the liquid by the external fluxes is of paramount importance. In absence of desorption from the liquid, refill scales linearly with time. In presence of desorption, we eschew considering time explicitly by considering the net refill (input minus desorption). This study obviously assumes that nucleation occurs, but we will also specify in which cases it does not. However, the kinetics of nucleation are not considered.

In the second part of the work (Section VI), we simplify the description of the system but we lift the restriction to fast kinetics. We introduce time explicitly, and in particular the two time scales of delivery/attachment to the ML and system refill. We describe analytically the ML growth kinetics as a function of the competition between these processes. The whole ML growth cycle can then be treated in a unified fashion, without distinguishing $a$ priori between stages 1 and 2 (although this approach allows us to find out when this distinction is legitimate).

\section{DESCRIPTION AND ENERGETICS OF THE MONOLAYER-LIQUID SYSTEM}

\section{A. Introduction}

We consider the case of binary III-V NWs grown with or without a foreign metal catalyst $m$, such as gold. To 
investigate the energetics of ML formation, we calculate the variation $\Delta G_{0 i}$ of the Gibbs free energy of the system $\mathrm{NW}+$ droplet between nucleation $(t=0)$ and formation of a fractional ML made of a number $i$ of III-V pairs, for $0 \leq i \leq i_{\mathrm{ML}}$ (with $i_{\mathrm{ML}}$ the number of pairs in a complete $\mathrm{ML}$ ), i.e. for coverages $\theta=i / i_{\mathrm{ML}}$ between 0 and 1 .

When nucleation occurs in a bulk mother phase, the classical nucleation theory indicates that $\Delta G_{0 i}=$ $-\Delta \mu i+G_{e}(i)$, with $\Delta \mu>0$ the difference of chemical potential per III-V pair between liquid and solid and $G_{e}(i)$ the energy of the edge of the ML. Since the fraction of atoms removed from the liquid to form the ML is negligible, $\Delta \mu$ remains constant and, for unrestricted growth of a ML of fixed shape, the edge length varies as $i^{1 / 2}$. Therefore, $\Delta G_{0 i}$ first increases with $i$, culminates at the nucleation barrier and then decreases continually as soon as the linear term takes over.

For a nanodroplet, the numbers of atoms of the different species, in particular that of group V, are so small that transferring atoms from liquid to solid alters the atomic concentrations and hence $\Delta \mu[2,20,22,25]$. At stage 1 , in absence of liquid refilling, this depletion gradually reduces $\Delta \mu$ and makes the transfer less and less favorable. A point may be reached were transfer becomes inhibited. On the other hand, the edge contribution $G_{e}$ is affected by the fact that the ML progresses at the solidliquid interface, which is both finite and bordered by a triple phase line (TPL), and the ML shape changes vastly with coverage [15]. The implications for growth kinetics have not been discussed so far, apart of course for the impact of the TPL upon nucleation [27]. It appears necessary to treat these two points jointly.

\section{B. General formulae}

All calculations are performed at a given temperature $T$ and the temperature dependence of the various quantities considered is kept implicit. The state of the bulk liquid is defined by the atomic concentrations of two species, e.g. those of the group III and V semiconductor elements, $c_{3}$ and $c_{5}$, the concentration of the catalyst being $c_{m}=1-c_{3}-c_{5}\left(c_{m}=0\right.$ for self-catalyzed growth $)$. The chemical potentials $\mu_{k}$ of the three species depend only on these concentrations and can be expressed as a function of the excess Gibbs energy of the liquid, $E_{m}^{L}\left(c_{3}, c_{5}\right)$, which quantifies the difference between actual and ideal liquids $[28,29]$ :

$$
\begin{aligned}
\mu_{3}= & { }^{0} \mu_{3}+E_{m}^{L}+\left(1-c_{3}\right) \frac{\partial E_{m}^{L}}{\partial c_{3}}-c_{5} \frac{\partial E_{m}^{L}}{\partial c_{5}} \\
& +k_{B} T \ln c_{3} \\
\mu_{5}= & { }^{0} \mu_{5}+E_{m}^{L}-c_{3} \frac{\partial E_{m}^{L}}{\partial c_{3}}+\left(1-c_{5}\right) \frac{\partial E_{m}^{L}}{\partial c_{5}} \\
& +k_{B} T \ln c_{5}
\end{aligned}
$$

$$
\begin{aligned}
\mu_{m}= & { }^{0} \mu_{m}+E_{m}^{L}-c_{3} \frac{\partial E_{m}^{L}}{\partial c_{3}}-c_{5} \frac{\partial E_{m}^{L}}{\partial c_{5}} \\
& +k_{B} T \ln \left(1-c_{3}-c_{5}\right)
\end{aligned}
$$

with $k_{B}$ the Boltzmann constant and ${ }^{0} \mu_{k}$ the chemical potential of pure liquid $k$ at temperature $T$.

Since the system evolves by transfer of III-V pairs from liquid to the solid ML and, at stages 2 and 3, from vapor to liquid, we have:

$$
\Delta G_{0 i}=G_{S}(i)+G_{L}^{(i)}-G_{L}^{(0)}+\Delta G_{V}^{(i)}
$$

where $G_{S}$ is the free energy of formation of the solid island, which only depends on the number $i$ of pairs that it contains, $G_{L}^{(i)}$ the Gibbs free energy of the liquid in state $(i)$, at which the ML has reached the size of $i$ III-V pairs. For a given initial state, it is only at stage 1 that the state of the liquid depends solely on number $i$; at stages 2 and 3 , it is also affected by refill. Finally, $\Delta G_{V}^{(i)}$ is the change of Gibbs energy of the vapor.

The formation energy of the solid ML comprises the two usual terms related to its volume and its edge, respectively:

$$
G_{S}(i)=i \mu_{S}+G_{e}(i)
$$

with $\mu_{S}$ the chemical potential of a III-V pair in bulk solid (which depends only on temperature) and $G_{e}(i)$ the energy of formation of the island edge, to be discussed in Section II C.

The contribution of the liquid to Eq. (4) also comprises a term $\Delta G_{N}$ involving the numbers of atoms of the different species and their chemical potentials, and a term $\Delta G_{L V}$ reflecting possible variations of the outer area of the liquid droplet (liquid-vapor interface) between states 0 and $i$ :

$$
G_{L}^{(i)}-G_{L}^{(0)}=\Delta G_{N}+\Delta G_{L V}
$$

The first term is:

$$
\begin{aligned}
\Delta G_{N}= & N_{3}^{(i)} \mu_{3}^{(i)}+N_{5}^{(i)} \mu_{5}^{(i)}-N_{3}^{(0)} \mu_{3}^{(0)}-N_{5}^{(0)} \mu_{5}^{(0)} \\
& +N_{m}\left(\mu_{m}^{(i)}-\mu_{m}^{(0)}\right)
\end{aligned}
$$

where $N_{k}^{(j)}$ and $\mu_{k}^{(j)}$ are the numbers and bulk chemical potentials of the atoms of species $k$ (III, V, metal) in state $j$ (the number of metal atoms being constant, the state index is omitted). As mentioned above, due to the small size of the droplet, the atomic concentrations and hence the chemical potentials change because of the depletion of the liquid at stage 1 and because of droplet refill at stages 2 and 3 . The surface contribution $\Delta G_{L V}$ will be discussed in Sections II D and V.

The contribution of the vapor to Eq. (4) is null at stage 1 and equal to $-j_{3} \mu_{3}^{V}-j_{5} \mu_{5}^{V}$ at stages 2 or 3 , with $\mu_{k}^{V}$ the chemical potential of species $k$ in the vapor and $j_{k}$ the net number of atoms of species $k$ transferred from the vapor to liquid or solid. 


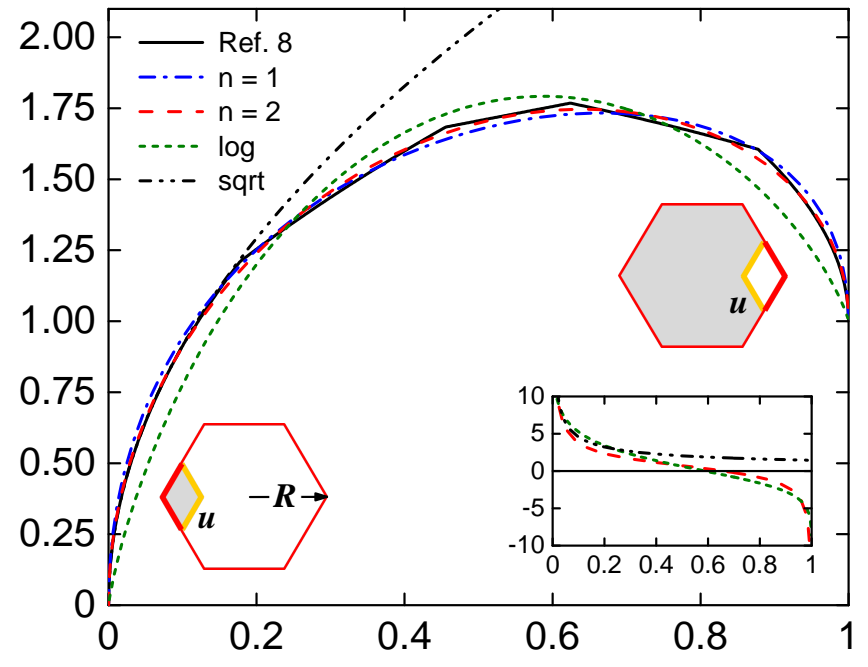

FIG. 2. Variation with ML coverage of the normalized ML edge energy. Composite full black curve: Fit to the experiments obtained in ref. [15]. Dash-dot blue curve: Eq. (8) with $n=1, A \simeq 3.3748, B \simeq 2.2993, a_{0}=-B, a_{1}=1-A+B$. Dash red curve: The same with $n=2, A \simeq 3.0231, B \simeq$ 1.9475, $a_{0}=-B, a_{1}=1-A+B-a_{2}, a_{2} \simeq-0.6746$. Short dash green curve (log): Fit by function $G_{e}^{L}$, with $A \simeq 2.4301, B \simeq$ 1.2193. Dash-dot-dot black curve (sqrt): Function $G_{e}^{u}=A^{\prime} \theta^{1 / 2}$, with $A^{\prime}$ adjusted to reproduce experimental fit at low coverage. Schematics of the partial ML (grey area) at low and high coverages, with thick red and orange lines marking ML edge portions at TPL (red hexagon) and away from TPL, respectively. Insert: Variations of the derivatives of the normalized edge energy with respect to $\theta$ for fits $n=2$, "log" and "sqrt" (same line types).

\section{Monolayer edge energy}

In a recent work dealing with the formation of individual MLs in wurtzite (WZ) Au-catalyzed GaAs NWs [15], we showed that, whatever the coverage $\theta$, the edge of the ML is composed of three types of segments with well-defined crystalline orientations, one located at the triple phase line (TPL) that limits the NW hexagonal top facet and two away from the TPL. In particular, the first observable island is located at the TPL (and indeed at a corner of the TPL). The evolution with coverage of the ML edge configuration could be reproduced by assigning nearly identical specific areal energies $\gamma_{1}, \gamma_{2}$ to the two inner step segments (these energies are assumed to be unaffected by the variations of liquid composition during ML growth) and a much lower energy $\gamma_{0}$ to the segment at the TPL (Fig. 2, composite curve). We argued that $\gamma_{0}$ is actually an effective energy equal to the difference between those of (i) that part of the ML edge in contact with the vapor, at the TPL (this energy is usually equaled to the sidewall energy) and (ii) the portion of liquid surface deleted when material from the liquid is added to the ML at the TPL. This extends our original interpretation of WZ formation by nucleation at the TPL
[27] to the later stages of ML formation.

The edge energy thus modelled [15] varies non monotonically with coverage (Fig. 2, full composite curve). Based on our experiments and modelling, this variation (not discussed in Ref. [15]) can be understood as follows. At small coverage, where only one type of internal edge (of energy $\gamma_{1}$ ) is present, the ML is a rhombus of side $u$, area $\sqrt{3} u^{2} / 2$ and $G_{e}=2\left(\gamma_{0}+\gamma_{1}\right) h u$, with $h$ the ML height (Fig. 2, bottom left). The top facet of a NW of radius $R$ (measured from center to corner of the hexagon; see Fig. 2) has area $S_{H}=3 \sqrt{3} R^{2} / 2$, hence $G_{e}=2 \sqrt{3}\left(\gamma_{0}+\gamma_{1}\right) h R \theta^{1 / 2}$ (with $\theta=i / i_{\mathrm{ML}}$ ). This is the standard square root dependence upon the number of pairs for 2D islands, mentioned above. At high coverage (Fig. 2, right), the situation is reversed, with an uncovered area $\sqrt{3} u^{2} / 2$ and $G_{e}=\gamma_{0} h\left(P_{H}-2 u\right)+$ $2 \gamma_{1} h u$, with $P_{H}=6 R$ the TPL perimeter, hence $G_{e}=h R\left[6 \gamma_{0}+2 \sqrt{3}\left(\gamma_{1}-\gamma_{0}\right)(1-\theta)^{1 / 2}\right]$. These behaviors can be reproduced if the edge energy is assumed to vary as:

$$
G_{e}^{(n)}(i)=6 h R \gamma_{0}\left[A \theta^{1 / 2}+B(1-\theta)^{1 / 2}+P_{n}(\theta)\right]
$$

where $P_{n}(\theta)=\sum_{0}^{n} a_{p} \theta^{p}$ is a polynomial of degree $n$ such that $P_{n}(0)+B=0, P_{n}(1)+A=1$. Moreover, we then expect $A \approx\left(1+\alpha_{e}\right) /\left(\sqrt{3} \alpha_{e}\right)$ and $B \approx\left(1-\alpha_{e}\right) /\left(\sqrt{3} \alpha_{e}\right)$, with $\alpha_{e}=\gamma_{0} / \gamma_{1}$. With $\alpha_{e}=0.25$ [15], this gives $A \approx 2.89, B \approx 1.73$. Fig. 2 shows that, even for $n=1$ or $2, G_{e}^{(n)}$ follows well the variations of $G_{e}$ over the full range $0 \leq \theta \leq 1$, although the values of $A$ and $B$ need adjusted for the best overall fit. A logarithmic approximation, $G_{e}^{L}(i)=6 h R \gamma_{0}[-A \theta \ln \theta-B(1-\theta) \ln (1-\theta)+\theta]$, is also illustrated in Fig. 2.

Even though our previous work dealt with a specific Au-catalyzed GaAs NW, the smallest island that we observe always has part of its perimeter at the TPL, whatever the catalyst composition, including self-catalyzed growth. This suggests that the non-monotonic behavior of $G_{e}$ described by Eq. (8) is generic, although the values of the various constants involved may depend on the nature of the metal catalyst (if any) and group III element and on their concentrations in the liquid. We will therefore use these approximations whatever the catalyst composition.

\section{Surface energy of the droplet}

It is clear from Section II C that, as in Ref. [15], we take fully into account the hexagonal shape of the ML. The geometry of a liquid droplet resting wholly or partially on such a base has been calculated numerically in the past [30] and is still being investigated. Here, we intend to use the simple analytical formulas for a droplet in the shape of a spherical cap. This requires values of the uniform contact angle $\beta_{d}$ of such a droplet and of the radius of its circular base which we define as $R_{b}=\alpha_{b} R$, 
with $\alpha_{b}$ a constant close to 1 . Since the spherical shape and the uniformity of the contact angles are approximations, the choice of $\alpha_{b}$ is somewhat arbitrary but, reasonably, $\alpha_{b}$ should be taken close to 1 . For instance, $\alpha_{b}=3^{3 / 4} /(2 \pi)^{1 / 2} \approx 0.909$ for a circular base having the same area as the hexagon and $\alpha_{b}=3 / \pi \approx 0.955$ for equal perimeters.

The surface area of the droplet, and hence its energy, may vary for three reasons. First, if the volumes of a pair in liquid and solid are different, the transfer from liquid to solid modifies the volume enclosed by the liquid surface, and thus its area. This produces an energy variation $b i$, where $b=2 \gamma_{L V}\left(\omega_{S}-\omega_{L}\right) \sin \beta_{d} / R_{b}$, with $\omega_{\phi}$ the volume of a III-V pair in phase $\phi=L$ or $S[19,25,31]$. Because it scales with $i$, this Gibbs-Thomson term, which intervenes at stages 1 and 2, can conveniently be incorporated as a small modification of $\mu_{S}$ or $\Delta \mu$ [25]. It is usually small because the pair volumes in liquid and solid are close [19] but might become significant at very small NW radii. For GaAs NWs of radius $R=20 \mathrm{~nm}$, it is only on the order of $1 \mathrm{meV} /$ pair.

Second, part of the ML edge is located at the TPL (Section II C). We recalled that transferring pairs from liquid to ML thus deletes part of the droplet surface, which is replaced by the solid ML edge. However, this is accounted for by assigning to the portion of the ML located at the TPL an effective energy $\gamma_{0}$ obtained by subtracting the energy of the liquid surface eliminated from that of the portion of ML edge in contact with the vapor. Because of this, as long as the ML is incomplete (stages 1 and 2), the droplet outer surface should be taken not as the actual liquid surface but as the full spherical cap having its base underneath the growing ML, as marked by a thick red line in Fig. 1, where the evolution of the system during a ML cycle is outlined. This point will be further discussed in Section V.

Third, the surface of the droplet of course increases if extra atoms are added from the vapor, as happens at stages 2 and 3 . These atoms may end up up either in the liquid or in the ML, the difference between the two involving again factor $b$.

\section{STAGE 1}

\section{A. General results}

Considering stage 1 as instantaneous, the system only evolves by transfer of III-V pairs from liquid to solid. As regards surface energy, according to Section II D, $\Delta G_{L V}$ thus reduces to the usually small term $b i$. In addition, $N_{k}^{(i)}=N_{k}^{(0)}-i$ for $k=3,5$ so that:

$$
c_{k}^{(i)}=\frac{N_{k}^{(0)}-i}{N_{\mathrm{tot}}^{(0)}-2 i} \text { for } k=3,5, \quad c_{m}^{(i)}=\frac{N_{m}}{N_{\mathrm{tot}}^{(0)}-2 i}
$$

with $N_{\text {tot }}^{(0)}=N_{3}^{(0)}+N_{5}^{(0)}+N_{m}$ the total number of atoms in the liquid in state $(0)$. Provided the dependence of
$E_{m}^{L}$ upon the compositions is known, the chemical potentials can be calculated for any $i$ from Eqs. (1)-(3) and (9). $G_{L}^{(i)}-G_{L}^{(0)}$ is then obtained from Eq. (6)-(7) (with $\Delta G_{L V}=b i$ ) and $\Delta G_{0 i}$ from Eqs. (4), (5) and (8). Note that, without depletion, the chemical potentials remain constant and we recover the usual formula, namely $\Delta G_{0 i}=-i \Delta \mu+G_{e}(i)$, with $\Delta \mu$ the difference of chemical potential per III-V pair between liquid and solid.

In order to find the number of pairs transferred from the liquid to the growing ML at stage 1, we calculate the derivative of $\Delta G_{0 i}$ for given initial conditions $N_{3}^{(0)}, N_{5}^{(0)}, N_{m}$. We have:

$$
\frac{d \Delta G_{0 i}}{d i}=\mu_{S}+b+\frac{d G_{e}}{d i}+\frac{d \Delta G_{N}}{d i}
$$

and, from Eq. (7):

$$
\frac{d \Delta G_{N}}{d i}=-\left(\mu_{3}^{(i)}+\mu_{5}^{(i)}\right)+\delta_{N}
$$

with $\delta_{N}=\sum_{k=3,5, m} N_{k}^{(i)} d \mu_{k}^{(i)} / d i$. By using Eqs. (1)-(3) and (9), we readily find that $\delta_{N}=0$, which is nothing else than the Gibbs-Duhem relation [32] for our system at fixed temperature and pressure. Hence:

$$
\frac{d \Delta G_{0 i}}{d i}=-\Delta \mu^{(i)}+\frac{d G_{e}}{d i}
$$

with $\Delta \mu^{(i)}=\mu_{3}^{(i)}+\mu_{5}^{(i)}-\mu_{S}-b$ the difference of chemical potential per III-V pair between liquid and solid in state $(i)$ (minus the small constant term $b$ ). Since no specific form of the excess Gibbs energy was chosen, this result holds whatever the catalyst and the group III and $\mathrm{V}$ atoms. The extrema of $\Delta G_{0 i}$ are thus given by the simple condition $d G_{e} / d i=\Delta \mu^{(i)}$.

Depending on the variations of chemical potential and edge energy with coverage, several cases can be considered. To emphasize the effects of the depletion of the liquid phase and of the non-monotonic variation of the ML edge energy (Section II C, Fig. 2), we illustrate three of these cases in Fig. 3. We assume here that the content of the droplet is not only sufficient for nucleation to occur, but also amounts to more than one ML, so that we may a priori consider coverages extending from 0 to 1 during stage 1. In Section III B, we will discuss more specifically the suppression of nucleation and the important case where, when nucleation occurs, the droplet group V content is equivalent to less than one ML (stage 1 must then terminate before ML completion).

(1) For a large mother phase and unrestricted growth of the ML (this is not adapted to NWs, but will serve as a reference), $\Delta \mu^{(i)}$ remains independent of $i$ (Fig. 3, curve (A)) and $G_{e}$ varies as $i^{1 / 2}$ (curve (a)). Hence, $d \Delta G_{0 i} / d i$ is initially positive but cancels for some critical size $i^{\star}$, provided $\Delta \mu>\left(d G_{e} / d i\right)(\theta=1)$. For $i \geq i^{\star}, d G_{e} / d i$ keeps decreasing and $d \Delta G_{0 i} / d i$ remains negative. This yields a single extremum for $\Delta G_{0 i}$ at $i=i^{\star}$, namely a maximum, the nucleation barrier. 


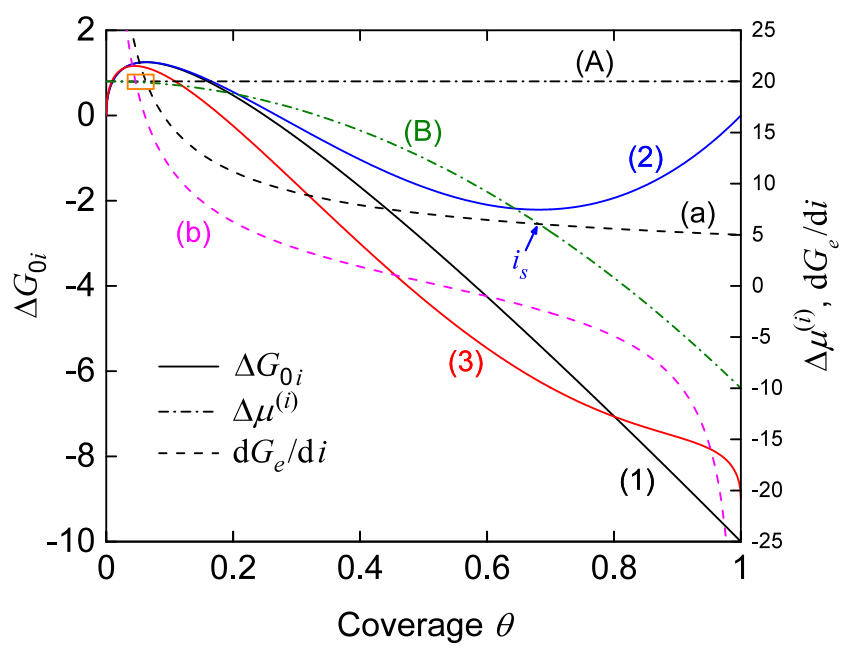

FIG. 3. Schematics of the variation of the work of formation $\Delta G_{0 i}$ of a ML with coverage (full curves, left scale) in three cases (numbers), depending on difference of chemical potential between liquid and solid, $\Delta \mu^{(i)}$ (dash-dotted lines, capitals, right scale: (A) constant; (B) affected by liquid depletion) and on dependence of ML edge energy $G_{e}$ upon ML size $i$, as illustrated by $d G_{e} / d i$ (dashed lines, small letters, right scale: (a) $G_{e}$ increasing as $i^{1 / 2}$; (b) non monotonic variation, as in Fig. 2). The three cases are: (1, black line) (A) and (a); (2, blue line) (B) and (a); (3, red line) (B) and (b). The extrema of $\Delta G_{0 i}$ correspond to the intersections of the $\Delta \mu^{(i)}$ and $d G_{e} / d i$ curves. The three maxima at left, all occurring within the orange rectangle, give critical size $i^{\star}$ in cases (1) to (3); the blue arrow marks the minimum occurring at $i=i_{s}$ in case (2). Units are arbitrary but consistent between left and right axes.

(2) It has long been recognized that, in the case of a mother phase small enough for the chemical potentials to be significantly affected by its depletion, the work of formation of a cluster may present a second extremum, now a minimum, for a certain size $i=i_{s}$ [7-12]. This occurs if $\Delta \mu^{(i)}$ (now curve (B)) decreases faster with $i$ than $d G_{e} / d i$ (curve (a)). At the two sizes $i^{\star}$ and $i=i_{s}$, the ML is in equilibrium, respectively unstable and stable, with the corresponding mother phase. Of course, the second extremum appears only if $i_{s} \leq i_{\mathrm{ML}}$. In the field of NWs, this was first considered by Dubrovskii [25]. Since extending the island beyond $i_{s}$ is energetically unfavorable (before droplet refill), we expect stage 1 to terminate at this point and call $i_{s}$ the stopping size. In the simple case where $i_{s}$ is large enough for $d G_{e} / d i$ to be negligible, this corresponds to $\Delta \mu^{(i)}=0$ (bulk liquid-solid equilibrium).

(3) The non-monotonic variation of $G_{e}$ (Section IIC) opens a third possibility, namely that the decrease of $\Delta \mu^{(i)}$ (curve (B)) is overcome by that of $d G_{e} / d i$ (curve (b)), which tends to $-\infty$ when $i \rightarrow i_{\mathrm{ML}}$ (Fig. 2 and Eq. (8)). Then, even if $\Delta \mu^{(i)}$ cancels or becomes negative, no minimum of $\Delta G_{0 i}$ is reached before ML completion. The decrease of the total energy of the step at high coverage (partly due to the decrease of its length) may thus allow the transfer of material from an undersaturated mother phase to the ML.

This surprising effect results from the non-monotonic size dependence of the energy of the boundary of the second phase, which itself results from the lower effective energy of the portion of this boundary located at the TPL (as compared to the portion located inside the droplet). In principle, it could thus occur in other cases of nucleation in a small mother phase, in particular if the boundary of the new phase tends to replace the external boundary of the mother phase. In the next section, we consider this question in more details, based on quantitative data available in the specific case of III-V NWs.

\section{B. III-V nanowires - Calculations and discussion}

For sake of simplicity, we use the notations pertaining to a cubic crystal of lattice parameter $a$. For growth along (111) and a hexagonal NW section of radius $R$ (Fig. 2), $h=a / \sqrt{3}$ and $i_{\mathrm{ML}}=6(R / a)^{2}$. Using Eq. (8) with $n=2$, we find, for $0 \leq \theta \leq 1$ :

$$
\frac{d G_{e}}{d i}=K\left[\frac{A}{2 \sqrt{\theta}}-\frac{B}{2 \sqrt{1-\theta}}+a_{1}+2 a_{2} \theta\right]
$$

with $K=a^{3} \gamma_{0} /(\sqrt{3} R)$.

For both metal-catalyzed and self-catalyzed growth, the group $\mathrm{V}$ concentration is much lower than that of any other component of the liquid. During ML formation, the chemical potential of the liquid is thus only affected by group $\mathrm{V}$ depletion [2] and the total number of atoms $N_{\text {tot }}$ can be taken as constant. The group V concentration $x$ and number of atoms $N_{5}$ in the liquid are then proportional: $x=N_{5} / N_{\text {tot }}$. The difference of chemical potential between liquid and solid (per III-V pair) at temperature $T$ can be approximated accurately by logarithmic function $k_{B} T \ln \left(x / x_{\mathrm{eq}}\right)$, with $k_{B}$ the Boltzmann's constant and $x_{\mathrm{eq}}$ the equilibrium concentration, which depends only on temperature for self-catalyzed growth and, more generally, on the concentrations of the other constituents $[18,19]$. Let us assume that nucleation occurs when $N_{5}^{(0)}=l_{0}$ group $\mathrm{V}$ atoms are present in the droplet. Then, during stage 1 :

$$
\Delta \mu^{(i)}=k_{B} T \ln \left(\frac{l_{0}-i}{l_{\mathrm{eq}}}\right)
$$

with $l_{\text {eq }} \simeq N_{\text {tot }} x_{\text {eq }}$. In addition, $N_{\text {tot }}=V_{d} / \omega_{L}$, with $\omega_{L}$ the average atomic volume of the liquid and $V_{d}$ the droplet volume. For our spherical droplet (Section II D), $V_{d}=f\left(\beta_{d}\right) R_{b}^{3}$, with $f$ the well-known function recalled, for instance, in Ref. [19]. Eq. (14) thus rewrites:

$$
\Delta \mu^{(i)}=k_{B} T \ln \left(M \frac{\theta_{0}-\theta}{x_{\mathrm{eq}}}\right)
$$

with:

$$
M=\frac{6 \omega_{L}}{\alpha_{b}^{3} f\left(\beta_{d}\right) a^{2} R}
$$


and $\theta_{0}=l_{0} / i_{\mathrm{ML}}$ the coverage equivalent to $l_{0}$, which may of course be larger than 1. On the other hand, Eq. (15) requires that $\theta \leq \theta_{0}$.

Whatever the value of $\theta_{0}, d G_{e} / d i$ and hence $d \Delta G_{0 i} / d i$ become infinite when $\theta \rightarrow 0$. If $\theta_{0}$ is low enough, nucleation may be prohibited. This depends on ML edge energy, as illustrated for $\theta_{0}=0.5$ in Fig. 4: nucleation is suppressed for $\gamma_{0}=0.2 \mathrm{~J} \mathrm{~m}^{-2}$, but not for $\gamma_{0}=0.03 \mathrm{~J} \mathrm{~m}^{-2}$. We do not consider this case further: we thus assume a first zero of $d \Delta G_{0 i} / d i$ for some coverage $\theta^{\star}=i^{\star} / i_{\mathrm{ML}}$.

If $\theta_{0}<1$, coverage is limited to $\theta_{0}$ and $\Delta \mu^{(i)} \rightarrow-\infty$ for $\theta \rightarrow \theta_{0}$. Since $d G_{e} / d i \rightarrow-\infty$ only for $\theta \rightarrow 1$, then $d \Delta G_{0 i} / d i \rightarrow \infty$ for $\theta \rightarrow \theta_{0}$ and $\Delta G_{0 i}$ must reach a minimum at some stopping size $i_{s}<l_{0}$, which cannot be suppressed, whatever the variation of $G_{e}$ with coverage (as illustrated in Fig. 4, curve $\theta_{0}=0.8$ ). This is similar to case $(2)$ discussed above for $\theta_{0}>1$.

If, on the other hand, the group $\mathrm{V}$ content of the droplet at nucleation is larger than the content of a $\mathrm{ML}\left(\theta_{0}>1\right), \theta$ may vary between 0 and 1 and several cases are possible (Fig. $4, \theta_{0}=1.1$ to 1.5 ). If $\theta_{0}$ is large enough, $\Delta \mu^{(i)}$ may remain larger than $d G_{e} / d i$ for $i^{\star} \leq i \leq i_{\mathrm{ML}}$, in which case there is no minimum for $\Delta G_{0 i}$ and a complete $\mathrm{ML}$ grows during stage 1, before refill (Fig. 4, curve $\theta_{0}=1.5$ ). For $\theta_{0}$ smaller (but larger than 1), we examine if, due to the nonmonotonic variation of $G_{e}$, the growth regime may shift from case $(2)$ (which pertains to monotonic $G_{e}$ ) to case (3). To this end, we compare the variations of $\Delta G_{0 i}$ for an hypothetical unrestricted ML growth (in which case $d G_{e} / d i=d G_{e}^{u} / d i=K A^{\prime} \theta^{-1 / 2} / 2$, as defined in the caption of Fig. 2; short orange dashes in Fig. 4) and for the non-monotonic case. Because $\Delta \mu^{(i)}$ and $d G_{e}^{u} / d i$ are respectively concave and convex functions of coverage, the condition for a minimum of $\Delta G_{0 i}$ to occur at incomplete coverage in the unrestricted case is that $\Delta \mu^{(i)}<d G_{e}^{u} / d i$ for $\theta=1$, or:

$$
k_{B} T \ln \left(M \frac{\theta_{0}-1}{x_{\mathrm{eq}}}\right)<\frac{K A^{\prime}}{2}
$$

This is more likely to occur for small $\theta_{0}-1$ and, given the expressions of $K$ and $M$, for large $\gamma_{0}$ and $x_{\mathrm{eq}}$, whereas the impact of $R$ and $T$ is not as clear (the latter because $x_{\text {eq }}$ increases with $T$ ).

We explored quantitatively the case of self-catalyzed WZ GaAs NWs, for which $x_{\text {eq }}$ can easily be calculated as a function of $T$ [19]; we use the parameters given in the caption of Fig. 2 to calculate $G_{e}^{(2)}(i)$ and $G_{e}^{u}(i)$ (Fig. 4). Due to the large number of free or unknown quantities involved $\left(\gamma_{0}, T, R, \beta_{d}, \alpha_{b}, \theta_{0}\right)$, we did not perform a systematic study. Nevertheless, we find that, generally, when $\theta_{0}>1, \Delta G_{0 i}$ does not reach a minimum for $\theta \leq 1$, so that a full ML tends to grow during stage 1 (recall that a minimum occurs if the curves representing $\Delta \mu^{(i)}$ and the appropriate $d G_{e} / d i$ intersect a second time). However, at relatively high growth temperature, and provided $\theta_{0}$ is not too large, such a minimum may be reached for

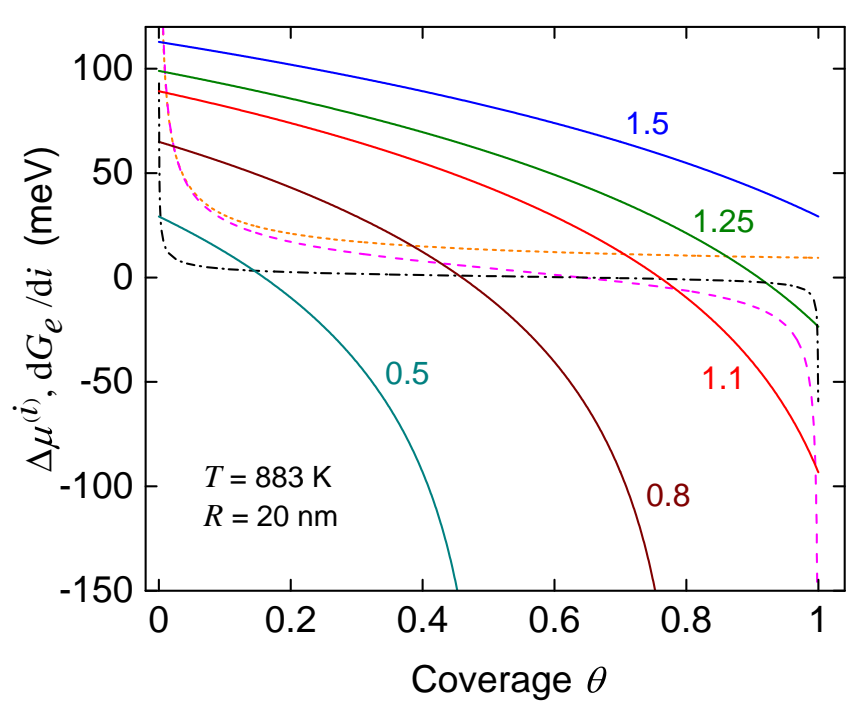

FIG. 4. Self-catalyzed WZ GaAs NWs of radius $R=20 \mathrm{~nm}$ growing at $T=883 \mathrm{~K}$. Variation with ML coverage of the difference of chemical potential per III-V pair between liquid and solid $\left(\Delta \mu^{(i)}\right.$, calculated with $b=0$ and $\alpha_{b}=3^{3 / 4} /(2 \pi)^{1 / 2}$, full curves) and of the derivative of the total ML edge energy with respect to ML size $i . \Delta \mu^{(i)}$ is shown for five different As contents of the liquid droplet at nucleation, expressed as equivalent coverage $\theta_{0}$ (indicated for each curve). The edge energy is taken as monotonic $G_{e}^{u}$ with $\gamma_{0}=0.2 \mathrm{~J} \mathrm{~m}^{-2}$ (short orange dashes) or non-monotonic $G_{e}^{(2)}$ with $\gamma_{0}=0.2 \mathrm{~J} \mathrm{~m}^{-2}$ (long magenta dashes) or $\gamma_{0}=0.03 \mathrm{~J} \mathrm{~m}^{-2}$ (black dash-dot curve).

both $G_{e}^{u}$ and $G_{e}^{(2)}$, albeit at different coverages (Fig. 4, $\theta_{0}=1.1$ ). Moreover, this minimum may disappear due to the non-monotonic variation of $G_{e}$, which amounts to switching from case (2) to case (3). This is shown in Fig. 4, where we observe that, for $\theta_{0}=1.25$, the maximum realized with $G_{e}^{u}$ vanishes with the non-monotonic $G_{e}$ for $\gamma_{0}=0.2 \mathrm{~J} \mathrm{~m}^{-2}$ (magenta dashes), albeit not for $\gamma_{0}=0.03 \mathrm{Jm}^{-2}$ (black dash-dot curve). The latter value corresponds to the specific energy $\gamma_{1}=0.123 \mathrm{Jm}^{-2}[19]$ and $\alpha_{e}=0.25[15]$.

\section{STAGE 2}

\section{A. Method}

During stage 2, which occurs if a partial ML of size $i_{s}$ has formed at stage 1 , the external fluxes refill the system with group III and group $\mathrm{V}$ atoms. The dynamics of ML extension for $i_{s} \leq i \leq i_{\mathrm{ML}}$ is linked to the partition of the newly provided atoms between ML and liquid.

In this section, we reason not in terms of time, but in terms of the net numbers of atoms that enrich either liquid or solid. This allows for some desorption from the liquid during the finite duration of stage 2 (and stage 3 ), which would make these net numbers smaller than the number of those entering the droplet from the vapor 
(note that desorption affects the group $\mathrm{V}$ species more than group III). However, desorption will only be taken explicitly into account in Section VI.

Let us thus assume that a net number $j$ of new group $\mathrm{V}$ atoms has been provided to the ML-liquid system. We quantify the partition of the $j$ atoms between liquid and ML by a single parameter $\chi$ : namely, the number of group $\mathrm{V}$ atoms added to the ML is $\chi j$, so that $i=i_{s}+\chi j$ while, in the liquid, $N_{5}^{(i)}=l_{0}-i_{s}+(1-\chi) j$. If the new atoms actually distribute between droplet and ML, then $0 \leq \chi \leq 1$. However, we allow $\chi$ to be larger than 1 , which corresponds to a refill-induced depletion of the liquid. According to our quasistatic approach, in order to determine the states of ML and liquid at a given refill level $j$, we search for a value of $\chi$ that minimizes $\Delta G_{0 i}$.

From Eq. (5) and since $d i / d \chi=j$,

$$
\frac{d G_{S}}{d \chi}=j\left(\mu_{S}+\frac{d G_{e}}{d i}\right)
$$

and, from Eq. (7):

$$
\frac{d \Delta G_{N}}{d \chi}=-j\left(\mu_{3}^{(i)}+\mu_{5}^{(i)}\right)+\sum_{k=3,5, m} N_{k}^{(i)} \frac{d \mu_{k}^{(i)}}{d \chi}
$$

From the Gibbs-Duhem relation, we find again that the right hand sum cancels.

By calculating the change of volume enclosed by the droplet surface between nucleation and state $(i)$, it is easily checked that $d \Delta G_{L V} / d \chi=b j$, even though desorption may affect differently the two atomic species. Finally, $\Delta G_{V}^{(i)}$ is non zero but independent of $\chi$. Gathering all contributions, we find, from Eq. (4), that $d \Delta G_{0 i} / d \chi=$ $j \delta$, with:

$$
\delta=-\Delta \mu^{(i)}+\frac{d G_{e}}{d i}
$$

In this expression, $\Delta \mu^{(i)}$ is calculated for $N_{5}^{(i)}$ group $\mathrm{V}$ atoms in the droplet and $d G_{e} / d i$ for $i$ pairs in the ML. These numbers, given above, both depend on $j$ and $\chi$. To express all numbers in terms of coverage, in addition to actual coverages $\theta_{s}=i_{s} / i_{\mathrm{ML}}$ (at end of stage 1 ) and $\theta=i / i_{\mathrm{ML}}$ (current), we define formally the coverages equivalent to refill level, $\theta_{j}=j / i_{\mathrm{ML}}$, and to group $\mathrm{V}$ liquid content, $\theta_{L}=N_{5}^{(i)} / i_{\mathrm{ML}}=\theta_{0}-\theta_{s}+(1-\chi) \theta_{j}$. We consider $\Delta \mu^{(i)}$ and $d G_{e} / d i$ as functions of $\theta_{L}$ and $\theta$, respectively, and $\delta$ as a function of $\theta_{j}$ and $\chi$. The constraints $\theta \leq 1(\mathrm{C} 1)$ for the $\mathrm{ML}$ and $\theta_{L} \geq 0(\mathrm{C} 2)$ for the liquid, set the maximum possible value $\chi_{m}\left(\theta_{j}\right)$ of $\chi$ for a given refill.

\section{B. Evolution of the system during refill}

The end of stage $1\left(\theta_{j}=0\right)$ is defined by $\Delta \mu^{(i)}\left(\theta_{0}-\theta_{s}\right)=\left(d G_{e} / d i\right)\left(\theta_{s}\right) . \quad$ At refill $\theta_{j}$, $\delta\left(\theta_{j}, \chi=0\right)=-\Delta \mu^{(i)}\left(\theta_{0}-\theta_{s}+\theta_{j}\right)+\left(d G_{e} / d i\right)\left(\theta_{s}\right)$.
Since $\Delta \mu^{(i)}$ increases with $\theta_{L}$, then $\delta\left(\theta_{j}, \chi=0\right)<0$. This indicates that the ML extends right from the beginning of stage 2 .

We first examine the evolution of the system in the case $\theta_{0}<1$. Then, at the beginning of stage 2 , as long as $\theta_{j} \leq 1-\theta_{0}$, constraint (C2) is more stringent than (C1) and $\chi_{m}=1+\left(\theta_{0}-\theta_{s}\right) / \theta_{j}>1$, with $\lim _{\chi \rightarrow \chi_{m}} \delta=$ $+\infty$ (set by $\left.\Delta \mu^{(i)}\right)$. Together with $\delta\left(\theta_{j}, \chi=0\right)<0$, this proves that there is at least one minimum of $\Delta G_{0 i}$ $(\delta=0, d \delta / d \chi>0)$ for $\chi \in\left[0, \chi_{m}\right]$. A stronger conclusion can even be reached. Indeed, for $\chi=1, \theta_{L}=\theta_{0}-\theta_{s}$ and $\theta>\theta_{s}$; since $d G_{e} / d i$ decreases when $\theta$ increases (Fig. 2), then $\delta\left(\theta_{j}, \chi=1\right)<0$ and $\Delta G_{0 i}$ must reach a minimum for a certain value $\chi_{j}>1$. Hence, not only all new group $\mathrm{V}$ atoms are transferred to the growing ML but a fraction of the atoms previously present in the liquid also contribute to ML extension. System refill thus has the apparently paradoxical effect of additionally depleting the liquid.

Upon further system refill, when $\theta_{j}>1-\theta_{0}$, constraint $(\mathrm{C} 1)$ becomes more stringent than $(\mathrm{C} 2)$ and $\chi_{m}=$ $\left(1-\theta_{s}\right) / \theta_{j}$. When $\chi \rightarrow \chi_{m}, \Delta \mu^{(i)}$ remains finite whereas $d G_{e} / d i \rightarrow-\infty$, and hence also $\delta$. The existence of a zero of $\delta$ cannot be guaranteed anymore. Nevertheless, without loss of generality, we may assume that, up to some refill level $\theta_{j} \geq 1-\theta_{0}, \delta$ cancels in interval $\left[0, \chi_{m}\left(\theta_{j}\right)\right]$. Then, there must be at least two zeros of $\delta$, one of which, $\chi_{j}\left(\theta_{j}\right)$, corresponds to a minimum of $\Delta G_{0 i}(d \delta / d \chi>0)$. If there is a refill level $\theta_{j}^{\star} \leq \tilde{\theta}_{j}$ such that $\theta_{s}+\chi_{j}\left(\theta_{j}^{\star}\right) \theta_{j}^{\star}=1$, then the ML is completed at refill $\theta_{j}^{\star}$. If not, then, as $\theta_{j}$ reaches $\tilde{\theta}_{j}, \delta$ remains negative for $\chi \leq \tilde{\chi}_{m}$, with $\tilde{\chi}_{m}=\left(1-\theta_{s}\right) / \tilde{\theta}_{j}$, and tends to $-\infty$ for $\chi \rightarrow \tilde{\chi}_{m} . \Delta G_{0 i}$ is then minimum for $\chi=\tilde{\chi}_{m}$. This implies that, at refill $\tilde{\theta}_{j}$, the ML suddenly completes, the overall contribution of the liquid being $\left(\tilde{\chi}_{m}-1\right) \tilde{\theta}_{j}$.

On the other hand, when $\theta_{0}>1$, constraint $(\mathrm{C} 1)$ is always the more stringent and the system evolves as described above in the case $\theta_{0}<1, \theta_{j}>1-\theta_{0}$.

Figure 5 shows the variations of ML coverage $\theta$ and group $\mathrm{V}$ liquid content $\theta_{L}$ as a function of refill $\theta_{j}$ (which is proportional to time) for two ML edge energies $\gamma_{0}$, two growth temperatures $T$ and various group $\mathrm{V}$ liquid contents $\theta_{0}$ at nucleation. The NW radius is $R=20 \mathrm{~nm}$. We only illustrate conditions under which the ML is not completed at stage 1, which corresponds in the figure to the sudden increase of $\theta$ (to a value less than 1) and decrease of $\theta_{L}$ at $\theta_{j}=0$. The inserts give the variations of $\chi_{j}$, which remains larger than 1: this means that, whatever the refill $\theta_{j}$, all atoms provided so far during stage 2 have served to extend the ML, with an additional contribution $\left(\chi_{j}-1\right) \theta_{j}$ from atoms previously stored in the liquid.

At low growth temperature ( $T=713 \mathrm{~K}$, blue curves), the group $\mathrm{V}$ concentration of the bulk liquid at equilibrium with GaAs (indicated in the figure) is very low. At nucleation, the liquid composition suddenly drops close to this value and the overwhelming majority of group 


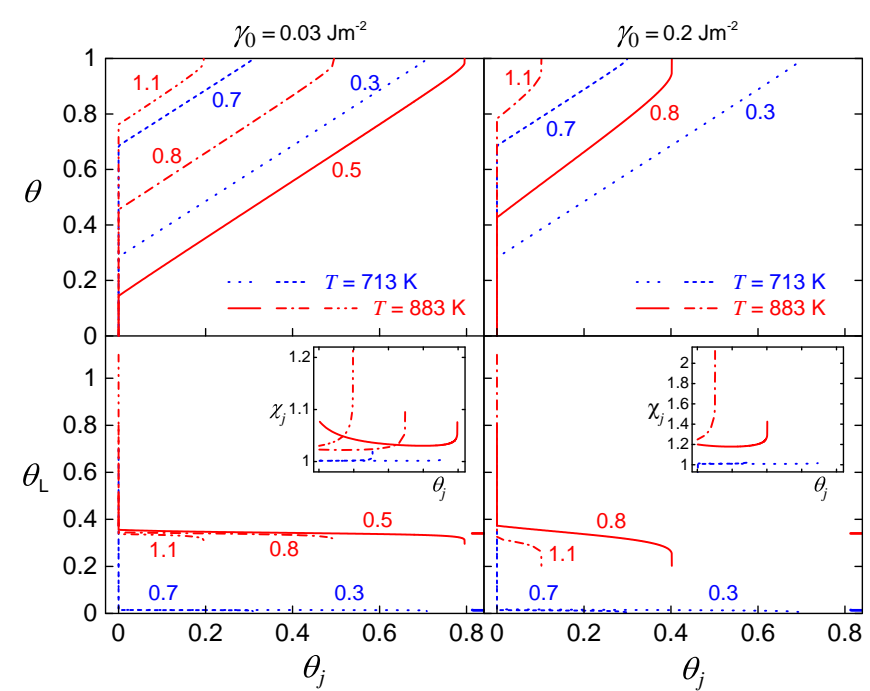

FIG. 5. Self-catalyzed WZ GaAs NWs of radius $R=20 \mathrm{~nm}$. Variations of ML coverage (top panels) and group V liquid content (bottom panels) as a function of refill, for edge energies $\gamma_{0}=0.03 \mathrm{~J} \mathrm{~m}^{-2}$ (left panels) and $0.2 \mathrm{~J} \mathrm{~m}^{-2}$ (right panels), growth temperatures $T=713 \mathrm{~K}$ (blue curves) and $883 \mathrm{~K}$ (red curves), and for various group $\mathrm{V}$ liquid content at nucleation, indicated in terms of ML $\left(\theta_{0}\right)$ near each curve. The short bars at the right of the bottom panels give the equilibrium concentrations of the bulk liquid with solid GaAs, at the two temperatures. The inserts show the variations with coverage of factor $\chi_{j}$ which determines the distribution between ML and liquid of the newly provided species (horizontal scales as in main panels). $\alpha_{b}$ as in Fig. 4.

$\mathrm{V}$ atoms present in the liquid serve to build the fractional ML. Subsequently, during stage $2, \chi_{j}$ remains very slightly larger than 1: ML extension is fed by the newly provided atoms with very little contribution from the liquid. Hence, ML coverage increases quasi-linearly, following the input, and liquid composition remains nearly constant. The ML fraction which grows suddenly at the end of stage 2 is minute. This holds for both edge energies considered.

At high growth temperature ( $T=883 \mathrm{~K}$, red curves), the bulk equilibrium group $\mathrm{V}$ liquid concentration represents a significant fraction of a ML, more than one third for the present NW radius. For $\gamma_{0}=0.03 \mathrm{~J} \mathrm{~m}^{-2}$, at nucleation, the group $\mathrm{V}$ concentration in the liquid falls very close to this value (due to the fact that $d G_{e} / d i \approx 0$, except for $\theta_{j}$ close to 0 or 1 ), and then decreases very slowly; accordingly, the ML coverage increases quasi-linearly. It is only at the very end of stage 2 that the coverage increases abruptly, thanks to the terminal depletion of the liquid. For the larger edge energy $\gamma_{0}=0.2 \mathrm{~J} \mathrm{~m}^{-2}$, the group $\mathrm{V}$ concentration in the liquid at stage 1 may be more different from its bulk equilibrium value (below or above, depending on the sign of $d G_{e} / d i$, as illustrated in the bottom right panel of Fig. 5), and it also decreases more significantly during stage 2 ; consequently, the ML coverage increases faster than input and slightly non-linearly. The terminal surge is also more marked.

\section{EFFECTIVE EDGE ENERGY}

At both stages 1 and 2, we used function $G_{e}^{(2)}$, derived from our previous work [15], to evaluate the energy of the edge of the ML. We stressed that, if we take the outer area of the droplet as fixed as long as no atom is added to the system (stage 1), or as increasing according to the number of atoms added (stage 2), which amounts to considering a whole spherical cap with base radius $R_{b}$ (red line in the four first four schematics of Fig. 1), this implies that the specific energy $\gamma_{0}$ of the portion of the edge located at the TPL is actually an effective energy. This energy corresponds to the difference between the actual energy of the edge-vapor interface and the energy of the droplet area deleted when atoms locate there to form part of the ML edge. This subtraction correctly cancels the energy of the virtual area added when considering the whole spherical cap.

This approach is admissible throughout stages 1 and 2, i.e. up to stage 2(a) in Fig. 1. After ML completion, the system should rather be described as at stages $2(\mathrm{~b})$ and 3 in this figure, with the droplet resting above the completed ML. This will restore for the next ML a configuration identical to that prevailing at beginning of stage 1. However, stages 2(a) and 2(b) represent the same state, namely the very end of stage 2 and simultaneous start of stage 3. For sake of consistency, it is thus essential that the droplet surface energies at 2(a) and 2(b) are equal. To verify this, we compare the changes of total surface energy between nucleation and stages 2(a) and 2 (b), which are respectively:

$$
\begin{aligned}
& \Delta G^{(a)}=\gamma_{0} P_{H} h+\Delta G_{L V}^{(a)} \\
& \Delta G^{(b)}=\gamma_{S V} P_{H} h+\Delta G_{L V}^{(b)}
\end{aligned}
$$

In each equation, the first term describes the change of ML edge energy and the second one the change of the surface energy of the liquid. The latter equals $\left(2 \sin \beta_{d} \gamma_{L V} / R_{b}\right) \Delta V^{(s)}$ (for $s=a, b$ ), with $\Delta V^{(s)}$ the change of the volume enclosed by this surface during stages 1 and 2. Noting that $\Delta V^{(a)}-\Delta V^{(b)}=S_{H} h$ and using the expressions of $P_{H}, S_{H}$ and $R_{b}$ given in Sections II C and II D, we find that the condition $\Delta G^{(a)}=\Delta G^{(b)}$ is equivalent to:

$$
\gamma_{0}=\gamma_{S V}-\frac{\sqrt{3}}{2 \alpha_{b}} \gamma_{L V} \sin \beta_{d}
$$

As noted before, $\alpha_{b}$ should be taken close to 1. Eq. (23) is thus very close to equation $\gamma_{\text {eff }}=\gamma_{S V}-\gamma_{L V} \sin \beta_{d}$ that we derived for TPL nucleation in the case of cylindrically symmetric NW and droplet, with $R_{b}=R$ [27]. The slight difference stems from the possible choice of $\alpha_{b} \neq \sqrt{3} / 2$ and from our combination of a spherical cap resting on an hexagonal facet, which is of course an approximation. The notion of an effective edge energy can 
thus be extended from nucleation [27] to the entire stage 1 (where, similarly, no atom is added to the liquid-ML system), whatever the ML fraction (as already noted by Dubrovskii [25]), but also to stage 2, where extra atoms are added to the system.

\section{KINETIC CALCULATIONS}

In this section, we present a fully analytical description of the ML growth cycle in the case where the edge energy contribution $d G_{e} / d i$ and its non-monotonous variations (Section II C) can be neglected. On the other hand, we treat the whole ML cycle in a unified fashion, without distinguishing a priori between a fast stage 1 (assumed to be instantaneous in Section III) and a slower stage 2 . Moreover, we take explicitly into account the desorption of group $\mathrm{V}$ atoms from the droplet (which was not required in Section IV, where we reasoned in terms not of time, but of the net number of vapor atoms added to the liquid-ML system). We retain notations $i$ for the number of III-V pairs in the fractional ML and $\theta$ for the corresponding ML coverage and introduce simplified notation $l$ for the current number $N_{5}^{(i)}$ of group $\mathrm{V}$ atoms in the liquid, consistent with notations $l_{0}$ and $l_{\text {eq }}$ for its initial and equilibrium values.

The kinetics are simply described by assuming that the ML growth rate depends linearly on the deviation between the current and bulk equilibrium group $\mathrm{V}$ contents of the liquid:

$$
\frac{d i}{d t}=\frac{l-l_{\mathrm{eq}}}{\tau}
$$

where $\tau$ is a certain characteristic time of island growth. The $l-l_{\text {eq }}$ term describes in a simplified fashion how the depletion of the liquid reduces the probability of incorporation to the growing ML.

At any time, the material balance has the form

$$
\frac{d l}{d t}=-\frac{d i}{d t}+v-v_{\mathrm{des}}
$$

showing that the total number of group $\mathrm{V}$ atoms in the droplet $l$ changes due to (i) island growth at rate $-d i / d t$, (ii) refill from vapor at rate $v$, and (iii) desorption of group $\mathrm{V}$ atoms from the droplet at rate $v_{\text {des }}$. Both $v$ and $v_{\text {des }}$ are proportional to the droplet surface area and hence to $R^{2}$. For the desorption term, we can use $v_{\text {des }}=v\left(l / l_{v}\right)^{2}$, which assumes that group $\mathrm{V}$ atoms desorb in the form of dimers (such as $\mathrm{As}_{2}$ or $\mathrm{P}_{2}$ ). Here, $l_{v} \propto v^{1 / 2}$ is the number of group $\mathrm{V}$ atoms in the droplet which corresponds to equilibrium with the vapor phase at a given flux $v$. This result follows from the general expression given in Ref. [19] if we keep only the logarithmic dependence of chemical potential on the group $\mathrm{V}$ concentration in the liquid. These considerations yield the material balance in the form

$$
\frac{d l}{d t}=-\frac{d i}{d t}+v\left[1-\left(\frac{l}{l_{v}}\right)^{2}\right]
$$

Eqs. (24) and (26) give the closed system for obtaining the growth kinetics in terms of island size $i(t)$ and liquid content $l(t)$, which should be solved with initial conditions $i(t=0)=0$ and $l(t=0)=l_{0}$ at nucleation.

Substitution of Eq. (24) into Eq. (26) results in the following closed equation for $l(t)$ :

$$
\frac{d l}{d t}=v+\frac{l_{\mathrm{eq}}}{\tau}-\frac{l}{\tau}-v\left(\frac{l}{l_{v}}\right)^{2}
$$

We first solve Eq. (27) for $l(t)$ and then use the result in Eq. (24) to obtain $i(t)$. The exact solutions can be presented in terms of coverages $\theta_{L}(t)$ and $\theta(t)$ in the form

$$
\begin{aligned}
\theta_{L}(t) & =\alpha \theta_{v} \frac{\theta_{0}+\beta \theta_{v}+\alpha \theta_{v} \tanh \left(\frac{\alpha}{\theta_{v}} \frac{t}{t_{\mathrm{ML}}}\right)}{\alpha \theta_{v}+\left(\theta_{0}+\beta \theta_{v}\right) \tanh \left(\frac{\alpha}{\theta_{v}} \frac{t}{t_{\mathrm{ML}}}\right)}-\beta \theta_{v} \\
\theta(t) & =2 \beta \theta_{v} \ln \left[\cosh \left(\frac{\alpha}{\theta_{v}} \frac{t}{t_{\mathrm{ML}}}\right)+\frac{\theta_{0}+\beta \theta_{v}}{\alpha \theta_{v}} \sinh \left(\frac{\alpha}{\theta_{v}} \frac{t}{t_{\mathrm{ML}}}\right)\right] \\
& -\left(\beta \theta_{v}+\theta_{\mathrm{eq}}\right) \frac{t}{\tau}
\end{aligned}
$$

with

$$
\begin{aligned}
\beta & =\frac{\theta_{v}}{2} \frac{t_{\mathrm{ML}}}{\tau} \\
\alpha & =\sqrt{1+\theta_{\mathrm{eq}} \frac{t_{\mathrm{ML}}}{\tau}+\beta^{2}}
\end{aligned}
$$

Here, $t_{\mathrm{ML}}=i_{\mathrm{ML}} / v$ is the time of deposition of one equivalent $\mathrm{ML}$ in absence of desorption and, in addition to equilibrium and equivalent initial coverages $\theta_{\text {eq }}$ and $\theta_{0}=\theta_{L}(t=0)$, we have introduced the coverage equivalent to desorption, $\theta_{v}=l_{v} / i_{\mathrm{ML}}$.

Equations (28) to (30) describe the kinetics of liquid content and ML coverage until the completion of the ML. A full ML forms at time $t_{g}$ such that $\theta\left(t_{g}\right)=1$. This $t_{g}$ can be called the ML growth time. After that, putting $d i / d t=0$ in Eq. (26), the liquid droplet refills according to:

$$
\theta_{L}(t)=\theta_{v} \tanh \left[\frac{t-t_{g}}{t_{\mathrm{ML}} \theta_{v}}+\operatorname{atanh}\left(\frac{\theta_{\min }}{\theta_{v}}\right)\right]
$$

with $\theta_{\min }=\theta_{L}\left(t_{g}\right)$ the equivalent liquid coverage at $\mathrm{ML}$ completion. This equation applies from $t_{g}$ to $t_{c}$, with $t_{c}$ the duration of the full ML growth cycle including refill to the initial $\theta_{0}$, defined by $\theta_{L}\left(t_{c}\right)=\theta_{0}$ if, neglecting for simplicity the nucleation-induced fluctuations, we assume a strictly periodic process.

These exact solutions require only the neglect of the edge term in the formation energy. Eqs. (28)-(29) describe in a unified fashion the entire ML growth $(0 \leq$ $\theta \leq 1)$ without distinguishing two stages as in Sections III and IV. They do not use the time scale hierarchy [26] $t / t_{\mathrm{ML}}<<1$ corresponding to very fast island growth to the stopping size in stage 1.

Let us now see what happens when this time scale hierarchy is present, as observed in our in situ growth experiments on Au-catalyzed [15] GaAs NWs and earlier 
in the vapor-solid-solid growth of Si NWs [13], where an initial fast ML step propagation (stage 1) is observed, followed by a slower advance (stage 2 ). Then, from the asymptotes of Eqs. (28) and (30) at $\tau / t_{\mathrm{ML}} \rightarrow 0$, the liquid content $\theta_{L}$ quickly stabilizes at

$$
\theta_{\text {min }} \cong \theta_{\text {eq }}
$$

while, from the asymptotes of Eqs. (29) and (30) in the same limit, the ML coverage varies as

$$
\theta(t) \cong \theta_{0}-\theta_{\mathrm{eq}}+(1-\eta) \frac{t}{t_{\mathrm{ML}}}
$$

where $\eta=\left(\theta_{\text {eq }} / \theta_{v}\right)^{2}$ is the ratio of the desorption rate at liquid-solid equilibrium to the refill rate. Therefore, the fractional ML quickly reaches the stopping size $\theta_{s}=\theta_{0}-\theta_{\text {eq }}$ corresponding to the equilibrium liquid, and then grows at the rate of effective refill (equal to the rate of external refill diminished by desorption from the liquid at equilibrium), which is consistent with Eq. (32). This is indeed the regime found in our thermodynamic investigation (Sections III and IV) when the ML edge energy is low (Fig. 5, left pannels). Of course, in this case, the stopping size exists only when $\theta_{0}-\theta_{\text {eq }}<1$. From Eq. (33), the asymptotic growth time is:

$$
t_{g} \cong\left[1-\left(\theta_{0}-\theta_{\mathrm{eq}}\right)\right] \frac{t_{\mathrm{ML}}}{1-\eta}
$$

Parameter $\eta$ depends strongly on temperature. It is usually close to zero but may become significant at high growth temperature, especially for small droplet diameters, where desorption is enhanced by the Kelvin effect [19] (for instance, we estimate that $\eta \approx 0.45$ for selfcatalyzed NWs of radius $7 \mathrm{~nm}$ at $T=873 \mathrm{~K}$ ). Otherwise, $t_{g}$ is only weakly affected by desorption. On the other hand, the refill (or waiting) time $t_{r}=t_{c}-t_{g}$ is easily obtained from Eq. (31) at $\theta_{\min }=\theta_{\text {eq }}$ :

$$
t_{r} \cong \theta_{v}\left[\operatorname{atanh}\left(\frac{\theta_{0}}{\theta_{v}}\right)-\operatorname{atanh}\left(\frac{\theta_{\mathrm{eq}}}{\theta_{v}}\right)\right] t_{\mathrm{ML}}
$$

and equals $\left(\theta_{0}-\theta_{\text {eq }}\right) t_{\mathrm{ML}}$ only in the absence of desorption $\left(\theta_{v} \rightarrow \infty\right)$. In the presence of desorption, the ML growth cycle $t_{c}$ can be noticeably longer than $t_{\mathrm{ML}}$. As a result, in the presence of the time scale hierarchy $\tau / t_{\mathrm{ML}}<<1$, the ML growth is almost insensitive to desorption because all atoms brought to the droplet from vapor are consumed by the growing ML. After the ML is completed, the refill depends strongly on desorption and is longer for higher desorption rates (or for smaller $\theta_{v}$ ).

In the absence of desorption $\left(\theta_{v} \rightarrow \infty\right)$, Eqs. (28) to (30) are reduced to

$$
\begin{gathered}
\theta_{L}(t)=\theta_{\mathrm{eq}}+\frac{\tau}{t_{\mathrm{ML}}}+\left(\theta_{0}-\theta_{\mathrm{eq}}-\frac{\tau}{t_{\mathrm{ML}}}\right) \mathrm{e}^{-t / \tau} \\
\theta(t)=\left(\theta_{0}-\theta_{\mathrm{eq}}-\frac{\tau}{t_{\mathrm{ML}}}\right)\left(1-\mathrm{e}^{-t / \tau}\right)+\frac{t}{t_{\mathrm{ML}}}
\end{gathered}
$$

These general solutions in the absence of desorption describe different growth regimes depending on the parameter $\tau / t_{\mathrm{ML}}$. At very small $\tau / t_{\mathrm{ML}}$ (or $t_{\mathrm{ML}} / \tau>>1$ ), there is a distinct time scale separation between a fast ML growth to the stopping size (stage 1) and slow refill (stage 2), as observed in our in situ MBE experiments [15] and described in the first part of this work. As the value of $\tau / t_{\mathrm{ML}}$ increases, this distinction fades. The maximum value of $\tau / t_{\mathrm{ML}}$ that allows a whole ML to grow during a time less than $t_{\mathrm{ML}}$ (which is required in the absence of desorption) is $\theta_{0}-\theta_{\text {eq }}$. In this limit, we end up with a regime where the ML grows steadily at the rate of refill $\left(\theta=t / t_{\mathrm{ML}}\right)$ while the liquid composition does not change at all $\left(\theta_{L}=\theta_{0}\right)$. This is close to the observations of Ref. [14], where the ML progresses smoothly without distinction between stages 1 and 2, albeit with a waiting time in each cycle.

Using in Eq. (37) the definition $\theta\left(t_{g}\right)=1$ of the island growth time $t_{g}$, we find from Eq. (36) that the liquid composition at the end of ML growth is:

$$
\theta_{\min }=\theta_{L}\left(t_{g}\right)=\theta_{0}-1+\frac{t_{g}}{t_{\mathrm{ML}}}
$$

which simply expresses the partial compensation of consuming one ML by refill at rate $v$. After that, from Eq. (31), the liquid refills linearly with time according to

$$
\theta_{L}(t)=\theta_{\min }+\frac{t-t_{g}}{t_{\mathrm{ML}}}
$$

When $\tau / t_{\mathrm{ML}}<<1$, since $\mathrm{e}^{-t / \tau} \rightarrow 0$ during the whole ML growth, the liquid content quickly stabilizes at $\theta_{\text {eq }}+\tau / t_{\mathrm{ML}}$, which is slightly higher than the equilibrium value, and the island growth time is

$$
t_{g}=\tau+t_{\mathrm{ML}}\left[1-\left(\theta_{0}-\theta_{\mathrm{eq}}\right)\right]
$$

after which, the liquid refills according to

$$
\theta_{L}(t)=\theta_{\mathrm{eq}}+\frac{\tau}{t_{\mathrm{ML}}}+\frac{t-t_{g}}{t_{\mathrm{ML}}}
$$

In the same limit, the refill (stage 3 ) occurs between times $t_{g}$ and $t_{g}+t_{r}$, with a refill time equal to

$$
t_{r}=t_{\mathrm{ML}}\left(\theta_{0}-\theta_{\mathrm{eq}}\right)-\tau
$$

As expected in the absence of desorption, the total duration of the $\mathrm{ML}$ growth cycle, $t_{c}=t_{g}+t_{r}$, equals $t_{\mathrm{ML}}$.

Dimensional analysis of our formulas leads to the following conclusions. Assuming as a first approximation that the droplet size does not affect the concentrations of group $\mathrm{V}$ atoms at nucleation and at equilibrium and neglecting the Kelvin effect, the values of $\theta_{0}, \theta_{\text {eq }}$ and $\theta_{v}$ scale with the NW radius $R$, because all corresponding atom numbers $l$ are proportional to the droplet volume $R^{3}$ whereas $i_{\mathrm{ML}}$ is proportional to the top facet area, hence $R^{2}$. The ML deposition time $t_{\mathrm{ML}}$ is independent of $R$, because both $v$ and $i_{\mathrm{ML}}$ scale as $R^{2}$. Therefore, $\theta_{0}-\theta_{\text {eq }}$ is also proportional to $R$ and reaches unity at a certain $R_{m}$ where the stopping effect disappears. In this case, when $\tau / t_{\mathrm{ML}}<<1$, the ML growth becomes almost 

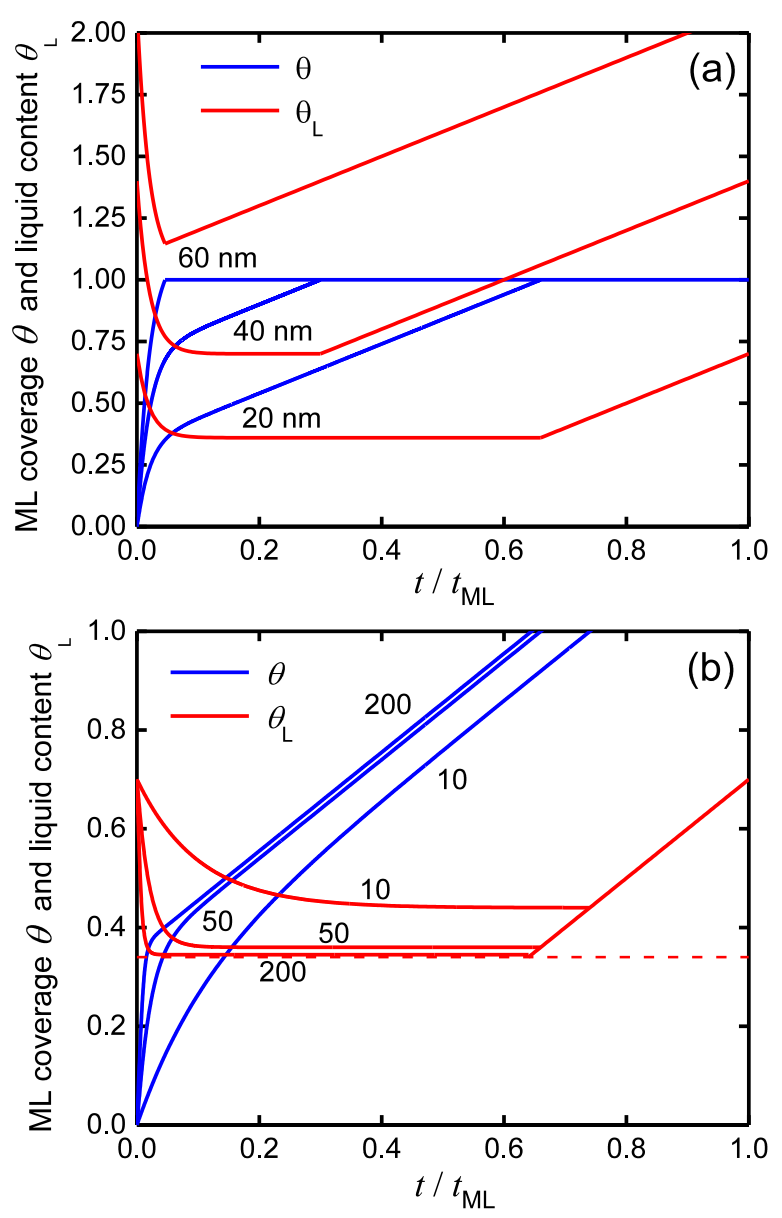

FIG. 6. (a) Evolution of $\theta$ and $\theta_{L}$ with normalized time $t / t_{\mathrm{ML}}$ in absence of group $\mathrm{V}$ desorption and at $t_{\mathrm{ML}} / \tau=50$, for three NW radii $R$ shown near each couple of curves. For $R=20 \mathrm{~nm}, \theta_{\text {eq }}=0.34$ and $\theta_{0}=0.7$, and we assume these coverages to scale with $R$, as discussed in the text. For $R=20 \mathrm{~nm}$ and $40 \mathrm{~nm}$, the ML grows faster at the beginning before reaching a stopping size, and then expands much slower at the rate of refill. The droplet content drops to near-equilibrium and stays constant in the slow ML growth stage. After that, $\theta_{L}$ increases linearly at the rate of refill. At $R=60 \mathrm{~nm}, \theta_{0}-\theta_{\text {eq }}=1.08>1$ and the stopping effect disappears: the ML covers almost instantaneously the whole facet and the liquid content never drops to equilibrium. (b) Same as (a) at a fixed NW radius of $20 \mathrm{~nm}$ for different $t_{\mathrm{ML}} / \tau$ shown near each curve. At a small $t_{\mathrm{ML}} / \tau$ of 10 , the stopping effect is not pronounced and $\theta_{L}$ drops to a minimum far above $\theta_{\text {eq }}$ (dashed line). At a large $t_{\mathrm{ML}} / \tau$ of 200 , there is a clear time scale hierarchy between the fast growth stage to a stopping size and slow growth at the rate of refill, while $\theta_{L}$ decreases almost instantaneously to equilibrium.

instantaneous, while the refill time is close to $t_{\mathrm{ML}}$ (without desorption) or $t_{c}>t_{\mathrm{ML}}$ (with desorption), as in the regime with $t_{g} / t_{r}<<1$ considered earlier [2, 20, 22, 31].

Figure 6(a) shows the evolution of the ML coverage and liquid content versus normalized time $t / t_{\mathrm{ML}}$ over a

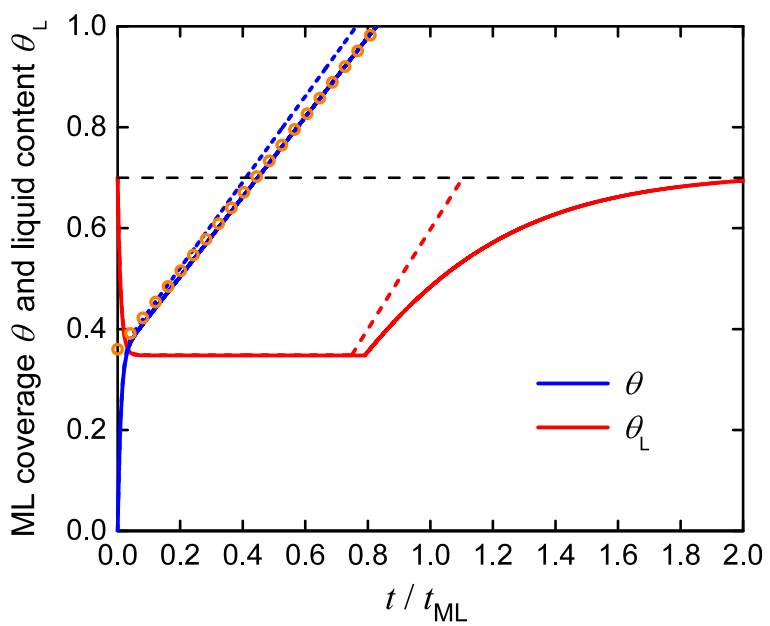

FIG. 7. Evolution of $\theta$ and $\theta_{L}$ with normalized time $t / t_{\mathrm{ML}}$ in presence of group $\mathrm{V}$ desorption at $\theta_{\text {eq }}=0.34, \theta_{0}=0.7$, $t_{\mathrm{ML}} / \tau=100, R=20 \mathrm{~nm}$, and two different $\theta_{v}=5$ (low desorption, dashed lines) and $\theta_{v}=0.71$ (high desorption, solid lines). The ML growth is almost identical in both cases. Refill takes slightly longer than in the absence of desorption at $\theta_{v}=$ 5 so that the initial liquid content at nucleation (indicated by horizontal dashed line) is recovered at $t \cong 1.1 t_{\mathrm{ML}}$. At $\theta_{v}=0.71$, refill is very long due to saturation of the $\theta_{L}$ curve, and the nucleation conditions are recovered only at $t \cong 2 t_{\mathrm{ML}}$. The line of circles shows the asymptote of $\theta$ given by Eq. (33) in the regime with high desorption.

ML cycle, obtained from Eqs. (36), (37) and (39) without desorption at $\theta_{\mathrm{eq}}=0.34$ (as in Fig. 5 , at the high temperature), $\theta_{0}=0.7$ at $R=20 \mathrm{~nm}, t_{\mathrm{ML}} / \tau=50$, and assuming $\theta_{0}-\theta_{\text {eq }} \propto R$ and $\theta_{\text {eq }} \propto R$, as discussed above, for different NW radii. The growth regime with a stopping size clearly occurs at $R=20 \mathrm{~nm}$ and $40 \mathrm{~nm}$. We observe a fast growth at the beginning, followed by a much slower growth at the rate of refill. The curves at $R=20 \mathrm{~nm}$ are quite similar to those shown in Fig. 5 (note that, in absence of desorption, $\theta_{j}=t / t_{\mathrm{ML}}$ ), but without the rapid changes of $\theta$ and $\theta_{L}$ at the end, caused by the edge energy effect. For $R=60 \mathrm{~nm}$, the VLS proceeds in the regime considered in the past $[2,20,22,31]$ with the ML growth being much faster than the refill of $\theta_{L}$. Of course, in this regime the liquid content never decreases to equilibrium. Figure $6(\mathrm{~b})$ shows the importance of the parameter $t_{\mathrm{ML}} / \tau$ (which was effectively considered as infinite in the previous sections) for observing the stopping effect. At a small $t_{\mathrm{ML}} / \tau$ of 10 , there is no clear resolution between the fast and slow growth stages, and $\theta_{L}$ remains far above equilibrium. At a large $t_{\mathrm{ML}} / \tau$ of 200 , the ML growth to a stopping size is almost instantaneous and this is followed by a slow growth at the rate of refill, while $\theta_{L}$ drops very quickly to equilibrium and stays at equilibrium until the ML is completed.

Figure 7 shows the evolution of the ML coverage and liquid content versus normalized time $t / t_{\mathrm{ML}}$ for the same parameters as in Fig. 6 at $R=20 \mathrm{~nm}$ and $t_{\mathrm{ML}} / \tau=100$, in the cases of low $\left(\theta_{v}=5\right)$ and high $\left(\theta_{v}=0.71\right)$ des- 
orption of group $\mathrm{V}$ atoms. It is seen that the entire ML growth stage is almost insensitive to desorption, as discussed above. Refill takes longer in both cases than without desorption and is much longer for high desorption. The initial liquid content at nucleation is recovered at $\theta_{j} \cong 1.1$ for low desorption and $\theta_{j} \cong 2$ for high desorption, meaning that the average $\mathrm{NW}$ growth rate is about $10 \%$ slower than the deposition rate for low desorption, and doubled for high desorption. As a general conclusion of this analysis, be can say that the ML growth can always be treated without desorption. The latter becomes important only during refill, which is much easier because no nucleation or growth occurs in this stage.

\section{SUMMARY AND CONCLUSIONS}

In this work, we investigated theoretically the formation of a ML during the vapor-liquid-solid growth of a $\mathrm{NW}$, when the concentration of one of the NW constituents in the liquid catalyst droplet is so low (on the order of one equivalent ML) that growing a single ML affects significantly the liquid phase. This is a common situation in the case for III-V NWs.

We first studied in details the energetics of ML formation in a quasistatic approximation. Assuming fast delivery and attachment of atoms from the liquid to the ML, we confirmed that, due to liquid depletion, the ML may reach a stopping size at which no further extension is possible before the system is refilled by the external growth fluxes. This first stage of growth is an instance of a theoretically well known phenomenon specific to systems of very small size. The stopping size corresponds to liquid-ML equilibrium and thus depends on the ML edge energy. Whereas this energy is usually assumed to increase with ML size, but only as its square root, so that its variations quickly become of minor importance, for a NW it varies non-monotonically, with a decrease during the second half of ML formation, becoming sharp before ML completion. We calculated and discussed the impact this has on the value and even the very existence of the stopping size. During the second stage of growth, the question arises of the partition of the newly provided atoms between liquid and growing ML. When the ML edge energy is low, the liquid retains during refill the composition close to bulk equilibrium reached in the first stage and the vast majority of new atoms join the ML.
If the edge energy is higher, its decrease tends to favor the incorporation of additional atoms initially present in the liquid, which may lead to a terminal surge in ML extension.

We then investigated the kinetics of ML formation, in the limit where the edge energy and its variations can be neglected. We performed fully analytical calculations based on a simple model that allows us not only to take into account jointly the two time scales of the problem, namely the external refill rate and the time of delivery and attachment to the ML, but also to include desorption of the sparse and volatile species (group $\mathrm{V}$ atoms) from the liquid. When the incorporation from the liquid to the ML is unhindered, we recover the two-stage description of our thermodynamic approach, with similar features. As incorporation gets slower, we describe a continuous transition from this pulsed regime to a smooth regime where the ML grows steadily at the rate of delivery from the vapor. The ML growth cycle comprises the actual growth of the full ML and the refill (or waiting) time before the nucleation of the next ML, during which the supersaturation of the liquid builds up again after depletion. We find that, in general, desorption affects little the time of formation of the ML but has a strong impact on the refill time.

\section{ACKNOWLEDGMENTS}

The authors acknowledge the support of the International Research Project "Physics of nanostructures and innovative devices based on compound semiconductors" (PHYNICS) established between Centre National de la Recherche Scientifique (CNRS) and the Russian Foundation for Basic Research. FG acknowledges financial support from Agence Nationale de la Recherche within project ESPADON ANR-15-CE24-0029 and from the European Commission within Marie Skodowska-Curie Innovative Training Network INDEED (No 722176). VGD thanks the Russian Foundation for Basic Research for financial support under grants Nos. 18-02-40006, 19-5253031, 20-52-16301 and 20-02-00351.

\section{REFERENCES}

[1] K. A. Dick, A review of nanowire growth promoted by alloys and non-alloying elements with emphasis on $\mathrm{Au}-$ assisted III-V nanowires, Prog. Cryst. Growth Charac. Mater. 54, 138 (2008).

[2] F. Glas, J. C. Harmand, and G. Patriarche, Nucleation antibunching in catalyst-assisted nanowire growth, Phys. Rev. Lett. 104, 135501 (2010).
[3] Y.-C. Chou, W.-W. Wu, S.-L. Cheng, B.-Y. Yoo, N. Myung, L. J. Chen, and K. N. Tu, In-situ TEM observation of repeating events of nucleation in epitaxial growth of nano $\mathrm{CoSi}_{2}$ in nanowires of $\mathrm{Si}$, Nano Lett. 8 , 2194 (2008).

[4] J. A. Elliott, Y. Shibuta, H. Amara, C. Bichara, and E. C. Neyts, Atomistic modelling of CVD synthesis of carbon nanotubes and graphene, Nanoscale 5, 6662 (2013). 
[5] K. Reyes, P. Smereka, D. Nothern, J. Mirecki Millunchick, S. Bietti, C. Somaschini, S. Sanguinetti, and C. Frigeri, Unified model of droplet epitaxy for compound semiconductor nanostructures: Experiments and theory, Phys. Rev. B 87, 165406 (2013).

[6] W. Theis and R. M. Tromp, Nucleation in Si(001) homoepitaxial growth, Phys. Rev. Lett. 76, 2770 (1996).

[7] M. Rao and B. J. Berne, Nucleation in finite systems: theory and computer simulation, Astrophys. Space Sci. 65, 39 (1979).

[8] D. Reguera, R. K. Bowles, Y. Djikaev, and H. Reiss, Phase transitions in systems small enough to be clusters, J. Chem. Phys. 118, 340 (2003).

[9] Z. Kožíšek and P. Demo, Influence of vapor depletion on nucleation rate, J. Chem. Phys. 126, 184510 (2007).

[10] J. W. P. Schmelzer and A. S. Abyzov, Thermodynamic analysis of nucleation in confined space: Generalized Gibbs approach, J. Chem. Phys. 134, 054511 (2011).

[11] Ø. Wilhelmsen, D. Bedeaux, S. Kjelstrup, and D. Reguera, Superstabilization of fluids in nanocontainers, J. Chem. Phys. 141, 071103 (2014).

[12] T. Philippe, Nucleation and superstabilization in small systems, Phys. Rev. E 96, 032802 (2017).

[13] C.-Y. Wen, J. Tersoff, M. C. Reuter, E. A. Stach, and F. M. Ross, Step-flow kinetics in nanowire growth, Phys. Rev. Lett. 105, 195502 (2010).

[14] D. Jacobsson, F. Panciera, J. Tersoff, M. C. Reuter, S. Lehmann, S. Hofmann, K. A. Dick, and F. M. Ross, Interface dynamics and crystal phase switching in GaAs nanowires, Nature 531, 317 (2016).

[15] J.-C. Harmand, G. Patriarche, F. Glas, F. Panciera, I. Florea, J.-L. Maurice, L. Travers, and Y. Ollivier, Atomic step flow on a nanofacet, Phys. Rev. Lett. 121, 166101 (2018).

[16] C. B. Maliakkal, E. K. Mårtensson, M. U. Tornberg, D. Jacobsson, A. R. Persson, J. Johansson, L. R. Wallenberg, and K. A. Dick, Independent control of nucleation and layer growth in nanowires, ACS Nano 14, 3868 (2020).

[17] F. Panciera, Z. Baraissov, G. Patriarche, V. G. Dubrovskii, F. Glas, L. Travers, U. Mirsaidov, and J.-C. Harmand, Phase selection in self-catalysed GaAs nanowires, Nano Lett. 20, 1669 (2020).

[18] F. Glas, Chemical potentials for Au-assisted vaporliquid-solid growth of III-V nanowires, J. Appl. Phys.
108, 073506 (2010).

[19] F. Glas, M. R. Ramdani, G. Patriarche, and J.-C. Harmand, Predictive modeling of self-catalyzed III-V nanowire growth, Phys. Rev. B 88, 195304 (2013).

[20] F. Glas, Statistics of sub-Poissonian nucleation in a nanophase, Phys. Rev. B 90, 125406 (2014).

[21] N. V. Sibirev, M. V. Nazarenko, D. A. Zeze, and V. G. Dubrovskii, Modeling the nucleation statistics in vaporliquid-solid nanowires, J. Cryst. Growth 401, 51 (2014).

[22] F. Glas and V. G. Dubrovskii, Self-narrowing of size distributions of nanostructures by nucleation antibunching, Phys. Rev. Mater. 1, 036003 (2017).

[23] E. S. Koivusalo, T. V. Hakkarainen, M. D. Guina, and V. G. Dubrovskii, Sub-Poissonian narrowing of length distributions realized in Ga-catalyzed GaAs nanowires, Nano Lett. 17, 5350 (2017).

[24] T. Tauchnitz, Y. Berdnikov, V. G. Dubrovskii, H. Schneider, M. Helm, and E. Dimakis, A simple route to synchronized nucleation of self-catalyzed GaAs nanowires on silicon for sub-Poissonian length distributions, Nanotechnology 29, 504004 (2018).

[25] V. G. Dubrovskii, Refinement of nucleation theory for vapor-liquid-solid nanowires, Cryst. Growth Des. 17, 2589 (2017).

[26] V. G. Dubrovskii, Self-regulated pulsed nucleation in catalyzed nanowire growth, Phys. Rev. B 87, 195426 (2013).

[27] F. Glas, J. C. Harmand, and G. Patriarche, Why does wurtzite form in nanowires of III-V zinc blende semiconductors?, Phys. Rev. Lett. 99, 146101 (2007).

[28] J. M. Prausnitz, R. N. Lichtenhalter, and E. Gomes de Azevedo, Molecular thermodynamics of fluid-phase equilibria (3rd edition) (Prentice Hall, Upper Saddle River, 1999).

[29] F. Glas, Comparison of modeling strategies for the growth of heterostructures in III-V nanowires, Crystal Growth \& Design 17, 4785 (2017).

[30] P. Krogstrup, S. Curiotto, E. Johnson, M. Aagesen, J. Nygård, and D. Chatain, Impact of the liquid phase shape on the structure of III-V nanowires, Phys. Rev. Lett. 106, 125505 (2011).

[31] V. G. Dubrovskii, N. V. Sibirev, J. C. Harmand, and F. Glas, Growth kinetics and crystal structure of semiconductor nanowires, Phys. Rev. B 78, 235301 (2008).

[32] H. B. Callen, Thermodynamics (Wiley, New York, 1960). 